\title{
Some Remarks on the Watson Transformation and Mode Theory
}

\author{
Leslie A. Berry
}

\author{
Contribution From the Central Radio Propagation Laboratory, National Bureau of Standards, \\ Boulder, Colo.
}

\begin{abstract}
The Watson transformation, which is the basis of VLF mode theory, is reviewed. It is shown that the disappearance of the line integral ("continuous spectrum") depends only on the properties of the earth (for a homogeneous ionosphere). Thus, the integral is interpreted just as in the classical groundwave case, i.e., it represents the waves which have reentered the air after traveling through the earth.

The limitations of the second-order Debye approximations have been discussed vaguely before. Actual calculation in specific cases shows that the attenuation rate of the first mode is in error by 60 percent at $16.6 \mathrm{kc} / \mathrm{s}, 25$ percent at $10 \mathrm{kc} / \mathrm{s}$, and 15 percent at $8 \mathrm{kc} / \mathrm{s}$ when the Debye approximations are used for all wave functions.

It is not possible to deduce effective parameters for the homogeneous ionosphere from consideration of the attenuation rate alone. It is suggested that simultaneous consideration of attenuation rate and phase velocity will remove ambiguity and an example calculation is given for $10.2 \mathrm{kc} / \mathrm{s}$. The resulting model ionosphere is at a height of $65 \mathrm{~km}$ with $\left|\omega_{r}\right| \sim 1.2$ $\left(10^{5}\right)$.
\end{abstract}

\section{Introduction}

The electromagnetic field excited by an elementary dipole between a homogeneous, spherical earth and a concentric homogeneous, isotropic ionosphere is given exactly by a series of zonal harmonics. Although the series can be summed with a digital computer [Johler and Berry, 1962], it is usually transformed into a quickly convergent series of residues, or modes. This transformation was first performed by Watson [1917, 1918], but it was not used for theoretical calculations (except for the "groundwave") for many years. The Watson transformation was recently reviewed preparatory to writing a computer program to sum the modes, and clarification of some details resulted.

Section 2 outlines the solution with sufficient detail to support the remarks made in section 3.1 about the disappearance and interpretation of the line integral. The validity of the secondorder Debye approximation is discussed quantitatively in section 3.2 and the problem of prescribing the model of the ionosphere is considered in section 3.3.

\section{Watson Transformation}

Figure 1 shows the geometry of the model. The origin of a spherical coordinate system $(r, \theta, \phi)$ is at the center of the homogeneous earth of radius $a$. The ionosphere is a concentric spherical shell, extending from $r=d=a+h$ to infinity. The source is a vertical electric Hertzian dipole at $\theta=0, r=b(a \leq b \leq d)$, and the field is to be found at any point $(r, \theta, \phi)$.

The electromagnetic properties of the media are given by their wave numbers, $k_{i}$. In the earth $(r<a)$,

$$
k_{2}=\frac{\omega}{c} \sqrt{\epsilon_{2}-i \frac{\sigma_{2} \mu_{0} c^{2}}{\omega}}
$$

where $\omega=2 \pi f$, and $f$ is the frequency, $c$ is the speed of light, $\epsilon_{2}$ is the relative permittivity, and $\sigma_{2}$, the conductivity, of the earth. In the air $(a<r<d)$,

$$
k_{1}=\frac{\omega}{c} \eta
$$

1 This work was performed as part of NBS Project 8510-11-85111. 
where $\eta$ is the index of refraction of air, and in the model ionosphere $(r>d)$,

where

$$
k_{3}=\frac{\omega}{c} \sqrt{1-i \frac{\omega_{r}}{\omega}}
$$

$$
\omega_{r}=\frac{\omega_{N}^{2}}{\nu+i \omega} .
$$

$\nu$ is the electron collision frequency, and $\omega_{N}$ is the electron gyrofrequency $\left(\omega_{N}^{2} \simeq 3.18 \times 10^{9} \mathrm{~N}\right.$, where $N$ is the number of electrons per cubic centimeter).

The field components in the air $(a<r<d)$ are found from a scalar Hertz-Debye potential [Johler and Berry, 1962]

$$
\Pi=\frac{1}{k_{1}^{2} r b} \sum_{n=0}^{\infty}(2 n+1) P_{n}(\cos \theta)\left[b_{n} \zeta_{n}^{(2)}\left(k_{1} r\right)+c_{n} \psi_{n}\left(k_{1} r\right)+\left\{\begin{array}{l}
\zeta_{n}^{(2)}\left(k_{1} b\right) \psi_{n}\left(k_{1} r\right) \\
\zeta_{n}^{(2)}\left(k_{1} r\right) \psi_{n}\left(k_{1} b\right)
\end{array}\right\}\right] \begin{aligned}
& r<b \\
& r>b
\end{aligned}
$$

$P_{n}(\cos \theta)$ is the Legendre function,

and

$$
\zeta_{v}^{(m)}(z)=\sqrt{\frac{\pi z}{2}} H_{v+1 / 2}^{(m)}(z), \quad m=1,2,
$$

$$
\psi_{v}(z)=\sqrt{\frac{\pi z}{2}} J_{v+1 / 2}(z),
$$

where $H_{v+1 / 2}^{(m)}(z)$ and $J_{v+1 / 2}(z)$ are Hankel and Bessel functions. [Jahnke and Emde, 1945.] The constants $b_{n}$ and $c_{n}$ are found from the boundary conditions.

$$
b_{n}=D_{n}^{-1}\left|\begin{array}{cccc}
1 & \zeta_{n}^{(2)}\left(k_{1} b\right) \psi_{n}\left(k_{1} a\right) & \psi_{n}\left(k_{1} a\right) & 0 \\
\frac{k_{1}}{k_{2}} R_{n} & \zeta_{n}^{(2)}\left(k_{1} b\right) \psi_{n}^{\prime}\left(k_{1} a\right) & \psi_{n}^{\prime}\left(k_{1} a\right) & 0 \\
0 & \zeta_{n}^{(2)}\left(k_{1} d\right) \psi_{n}\left(k_{1} b\right) & \psi_{n}\left(k_{1} d\right) & \zeta_{n}^{(2)}\left(k_{3} d\right) \\
0 & \zeta_{n}^{(2) \prime}\left(k_{1} d\right) \psi_{n}\left(k_{1} b\right) & \psi_{n}^{\prime}\left(k_{1} d\right) & \frac{k_{1}}{k_{3}} \zeta_{n}^{(2) \prime}\left(k_{3} d\right)
\end{array}\right|
$$

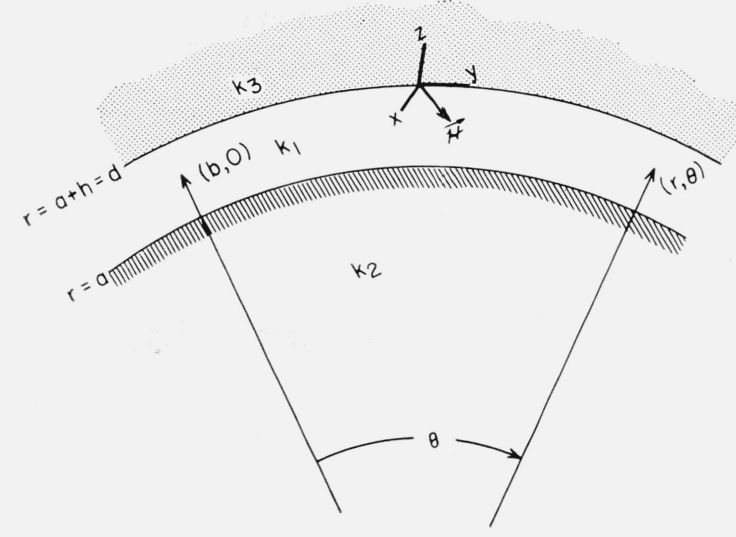

FiguRE 1. Geometry of the model.

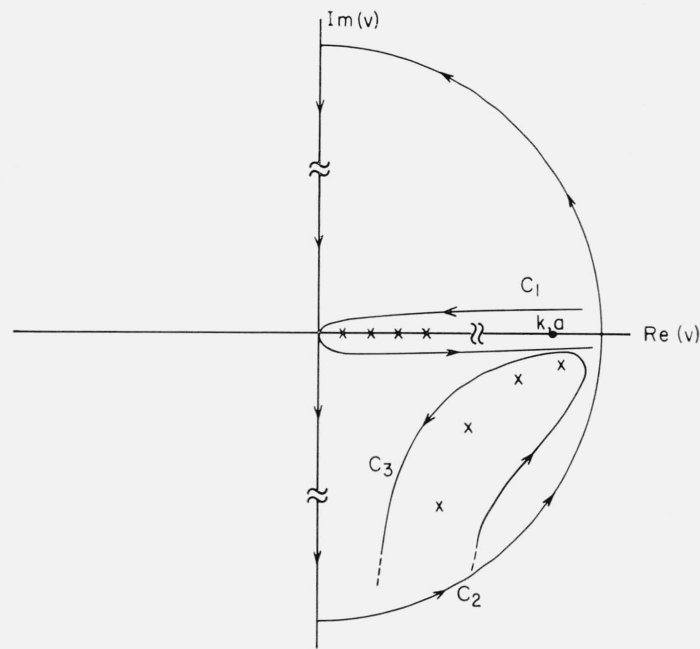

Figure 2. Complex v-plane showing contours of integration. 
where

and

$$
\psi_{n}^{\prime}(z)=\frac{d}{d z} \psi_{n}(z), \zeta_{n}^{(m) \prime}(z)=\frac{d}{d z} \zeta_{n}^{(m)}(z)
$$

$c_{n}$ is the same as $b_{n}$ except that the $\psi_{n}(x)$ functions in the third column of the determinant are replaced by $-\zeta_{n}^{(2)}(x)$ functions.

$$
D_{n}=\left|\begin{array}{cccc}
1 & \zeta_{n}^{(2)}\left(k_{1} a\right) & \psi_{n}\left(k_{1} a\right) & 0 \\
\frac{k_{1}}{k_{2}} R_{n} & \zeta_{n}^{(2) \prime}\left(k_{1} a\right) & \psi_{n}^{\prime}\left(k_{1} a\right) & 0 \\
0 & \zeta_{n}^{(2)}\left(k_{1} d\right) & \psi_{n}\left(k_{1} d\right) & \zeta_{n}^{(2)}\left(k_{3} d\right) \\
0 & \zeta_{n}^{(2) \prime}\left(k_{1} d\right) & \psi_{n}^{\prime}\left(k_{1} d\right) & \frac{k_{1}}{k_{3}} \zeta_{n}^{(2) \prime}\left(k_{3} d\right)
\end{array}\right|
$$

Using the substitutions,

and

$$
\psi_{n}(z)=\frac{1}{2}\left[\zeta_{n}^{(1)}(z)+\zeta_{n}^{(2)}(z)\right]
$$

$$
\zeta_{n}^{(m) \prime}(z)=\zeta_{n-1}^{(m)}-\frac{n}{z} \zeta_{n}^{(m)}(z),
$$

and after some algebra,

where

$$
b_{n} \zeta_{n}^{(2)}\left(k_{1} r\right)+c_{n} \psi_{n}\left(k_{1} r\right)=\frac{1}{2}\left[-\frac{A_{n}}{D_{n}}+\zeta_{n}^{(2)}\left(k_{1} b\right) \zeta_{n}^{(2)}\left(k_{1} r\right)\right],
$$

$$
A_{n}=\frac{1}{2}\left|\begin{array}{cccc}
1 & \zeta_{n}^{(2)}\left(k_{1} b\right) \zeta_{n}^{(1)}\left(k_{1} a\right) & \zeta_{n}^{(2)}\left(k_{1} r\right) \zeta_{n}^{(1)}\left(k_{1} a\right)-\zeta_{n}^{(1)}\left(k_{1} r\right) \zeta_{n}^{(2)}\left(k_{1} a\right) & 0 \\
\frac{k_{1}}{k_{2}} R_{n} & \zeta_{n}^{(2)}\left(k_{1} b\right) \zeta_{n}^{(1) \prime}\left(k_{1} a\right) & \zeta_{n}^{(2)}\left(k_{1} r\right) \zeta_{n}^{(1) \prime}\left(k_{1} a\right)-\zeta_{n}^{(1)}\left(k_{1} r\right) \zeta_{n}^{(2) \prime}\left(k_{1} a\right) & 0 \\
0 & \zeta_{n}^{(2)}\left(k_{1} d\right) \zeta_{n}^{(1)}\left(k_{1} b\right) & \zeta_{n}^{(2)}\left(k_{1} r\right) \zeta_{n}^{(1)}\left(k_{1} d\right)-\zeta_{n}^{(1)}\left(k_{1} r\right) \zeta_{n}^{(2)}\left(k_{1} d\right) & \zeta_{n}^{(2)}\left(k_{3} d\right) \\
0 & \zeta_{n}^{(2) \prime}\left(k_{1} d\right) \zeta_{n}^{(1)}\left(k_{1} b\right) & \zeta_{n}^{(2)}\left(k_{1} r\right) \zeta_{n}^{(1) \prime}\left(k_{1} d\right)-\zeta_{n}^{(1)}\left(k_{1} r\right) \zeta_{n}^{(2) \prime}\left(k_{1} d\right) & \frac{k_{1}}{k_{3}} \zeta_{n}^{(2) \prime}\left(k_{3} d\right)
\end{array}\right| .
$$

Then, from (4)

$$
\begin{aligned}
\Pi & =\frac{-1}{2 k_{1}^{2} r b} \sum_{n=0}^{\infty}(2 n+1) P_{n}(\cos \theta)\left[\frac{A_{n}}{D_{n}}-\zeta_{n}^{(2)}\left(k_{1} b\right) \zeta_{n}^{(2)}\left(k_{1} r\right)\right] \quad r \gtrless b, \\
& =\frac{-1}{2 k_{1}^{2} r b} \sum_{n=0}^{\infty}(2 n+1) P_{n}(\cos \theta) g(n) .
\end{aligned}
$$

Now

$$
\Pi=\frac{-i}{2 k_{1}^{2} r b} \int_{C_{1}} \frac{v P_{v-1 / 2}(-\cos \theta)}{\cos v \pi} g\left(v-\frac{1}{2}\right) d v,
$$

where $C_{1}$ is a contour enclosing the positive, real axis, as shown in figure 2. (All contours are taken counterclockwise.) Equation (16) reduces to (15) when the integral is evaluated by summing the residues at its poles, $v=n+\frac{1}{2}, n=0,1,2, \ldots$, noting that [Jahnke and Emde, 1945]

$$
P_{n}(-\cos \theta)=(-1)^{n} P_{n}(\cos \theta) .
$$

Consider the integral

$$
\int_{C_{2}} \frac{v P_{v-\frac{1}{2}}(-\cos \theta)}{\cos v \pi} g\left(v-\frac{1}{2}\right) d v=\int_{C_{2}} f(v) d v
$$

where $C_{2}$ is a semicircle in the right half-plane with infinite radius, and diameter along the 
imaginary axis, figure 2. $\quad P_{v-1 / 2}(-\cos \theta) / \cos v \pi$ decreases exponentially along the circumference of the semicircle so the integral along the circumference is zero [Watson, 1917]. Let $C_{3}$ be a contour in the right half-plane enclosing all zeroes of $D_{v-1 / 2}$ and no zeroes of $\cos v \pi$. Then

$$
\int_{+1 \infty}^{-1 \infty} f(v) d v=\int_{C_{2}} f(v) d v=\int_{C_{3}} f(v) d v+\int_{C_{1}} f(v) d v
$$

Substituting into (16),

where

$$
\begin{aligned}
\Pi & =\frac{i}{2 k_{1}^{2} r b}\left[\int_{C_{3}} f(v) d v+\int_{-1 \infty}^{1 \infty} f(v) d v\right] \\
& =\frac{-\pi}{k_{1}^{2} r b} \sum_{s=1}^{\infty} \frac{v_{s} P_{v_{s}-\frac{1}{2}}(-\cos \theta)}{\cos v_{s} \pi} \frac{A_{v_{s}-\frac{1}{2}}}{\left.\frac{\partial}{\partial v} D_{v-\frac{1}{2}}\right|_{v=v_{s}}}+\frac{i}{2 k_{1}^{2} r b} \int_{-i^{\infty}}^{+1 \infty} f(v) d v,
\end{aligned}
$$

$$
D_{v_{s-1 / 2}}=0, \operatorname{Re}(v)>0 .
$$

The terms in the residue series in (21) are called modes.

$$
\zeta_{-v-\frac{1}{2}}^{(m)}(z)=\exp \left[(-1)^{m+1} v \pi i\right] \zeta_{v-\frac{1}{2}}^{(m)}(z), \quad m=1,2
$$

[Jahnke and Emde, 1945]. Substituting (23) into (9) and (13), and looking at (15), it is seen that

if, and only if,

$$
\begin{gathered}
g\left(-v-\frac{1}{2}\right)=g\left(v-\frac{1}{2}\right) \\
R_{-v-\frac{1}{2}}\left(k_{2} a\right)=R_{v-\frac{1}{2}}\left(k_{2} a\right) .
\end{gathered}
$$

If the second order Debye approximation is used for the $\psi_{v}\left(k_{2} a\right)$ functions in (8)

$$
R_{v-\frac{1}{2}}\left(k_{2} a\right) \simeq i \sqrt{1-\left(\frac{v}{k_{2} a}\right)^{2}}
$$

which satisfies (25).

Cos $v \pi$ is an even function, $v$ is odd, and [Jahnke and Emde, 1945]

$$
P_{-v-\frac{1}{2}}(z)=P_{v-\frac{1}{2}}(z),
$$

so, to the extent that $(25)$ holds, $f(v)$ is an odd function and

$$
\int_{-1 \infty}^{+1 \infty} f(v) d v \cong 0
$$

The terms of the mode series are the residues of $f(v)$ at its poles $v_{s}$ in the fourth quadrant. Since $f(v)$ is an odd function of $v$ (to the extent that (26) holds), there is a corresponding set of poles $-v_{s}$ in the second quadrant. The Watson transformation could be carried out in the left half-plane and the field represented as the sum of the second-quadrant modes. This sum is equivalent to the sum of the fourth quadrant modes, both analytically and numerically.

\section{Discussion}

The important point in section 2 is the clear demonstration that only condition (25) is necessary for the line integral in (21) to vanish. Yet this apparently minor mathematical refinement throws considerable light on the physical interpretation.

\subsection{Significance of the Line Integral}

Wait has stated that the line integral in (21) vanishes if $\left|k_{3}\right|>>k_{1}$ [Wait, 1960], or if the fields satisfy the impedance boundary conditions at the surface of the ionosphere [Wait, 1963]. However (25) depends only on the radius and electromagnetic properties of the earth and is 
independent of the properties of the ionosphere. Actually, Friedman [1951] showed that the line integral is zero if the earth is perfectly conducting even if the atmosphere varies continuously in the radial direction.

Equation (26) becomes exact as $k_{2} a \rightarrow \infty$, so the integral in (21) is interpreted physically just as in the groundwave case, i.e., it represents the contribution of waves that have traveled through the earth and reentered the airspace. This interpretation was made explicit for the groundwave by Bremmer [1949] who wrote the integral as the sum of "rainbow terms." A ray that enters the spherical earth travels in a straight line through it (after the initial refraction) until it intersects the earth-air boundary, when some of its energy reenters the airspace. Thus, the integral in (21) is not quite zero, but since the waves attenuate rapidly inside the earth, physical reasoning shows that the integral is very small compared with the mode sum. Indeed, the approximation in (26) is in error no more than a few parts in $10^{5}$ for any practical VLF-ELF case, and is usually much less than that.

\subsection{Approximations to the Radial Wave Function}

Most of the early writers on mode theory, e.g. [Schumann, 1954; Bremmer, 1949; Wait, 1957, 1962b], used the second-order Debye approximations [Wait, 1957]

$$
\frac{\zeta_{v-1 / 2}^{(m)}(z)}{\zeta_{v-1 / 2}^{(m)}(z)} \cong(-1)^{m+1} i \sqrt{1-\left(\frac{v}{z}\right)^{2}}, \quad m=1,2,
$$

for the radial wave functions. Wait pointed out that (29) is not valid for functions of argument $k_{1} a$ for many cases of interest [Wait, 1960, 1962b], and since 1960, he has used a third-order approximation involving the Airey integral $w_{k}(x)$ [Fok, 1946; Wait, 1962b]. (See fig. 4.) This

approximation is the first term of a series in $\left(1-\left(\frac{v}{z}\right)^{2}\right)$ which represents the asymptotic form [Fok, 1946]

$$
H_{v}^{(m)}(z) \cong \frac{-i \xi \exp \left[(-1)^{m+1} \pi i / 6\right]}{\left(z^{2}-v^{2}\right)^{1 / 4}} H_{1 / 3}^{(m)}(-i \xi), \quad m=1,2
$$

where $\xi=v(\tanh w-w)$, and $\cosh w=v / z$. Figure 3 compares field computed with the Debye approximation at $16.6 \mathrm{kc} / \mathrm{s}$ [Wait, 1957 ] and at $1.6 \mathrm{kc} / \mathrm{s}$ [Wait and Carter, 1960] with fields computed using the more accurate (30).

The Debye approximation is apparently adequate at ELF, but is inadequate at $16.6 \mathrm{kc} / \mathrm{s}$. This inadequacy is more apparent if the curves are extended to greater distances, since the attenuation rate computed with the Debye approximation is only 60 percent of what it should be. Further computation shows that the attenuation rate of the first mode computed with the Debye approximation is in error by about 25 percent at $10 \mathrm{kc} / \mathrm{s}$ and by about 15 percent at $8 \mathrm{kc} / \mathrm{s}$.

\subsection{Selection of Ionospheric Model}

To make theoretical calculations of VLF field strengths, the effective height and electromagnetic parameters of the ionosphere (contained in the parameter $k_{3} d$ ) must be available. Figure 4 shows the attenuation rates of the more important modes as a function of electron density (or $\left|\omega_{r}\right|$ ) for $h=70 \mathrm{~km}$ and for frequencies of $25,10,4$, and $1 \mathrm{kc} / \mathrm{s}$. For a fixed frequency, as many as three values of $N$ give the same attenuation rate for the first mode. Indeed, Watson [1918] deduced a conductivity corresponding to an electron density of the order of several thousand electrons $/ \mathrm{cm}^{3}$; Wait [1957] deduced an electron density of about 600 electrons/ $\mathrm{cm}^{3}$, and Johler and Berry [1962] used a value of 56 electrons $/ \mathrm{cm}^{3}$. If the difference in frequency and assumed height are corrected for, these three models give about the same attenuation rate and are the three possible values mentioned above.

Figure 5 shows the phase velocity (relative to the speed of light) of the first mode as a function of electron density at 25,10 , and $4 \mathrm{kc} / \mathrm{s}$. The behavior of the phase velocity as a 


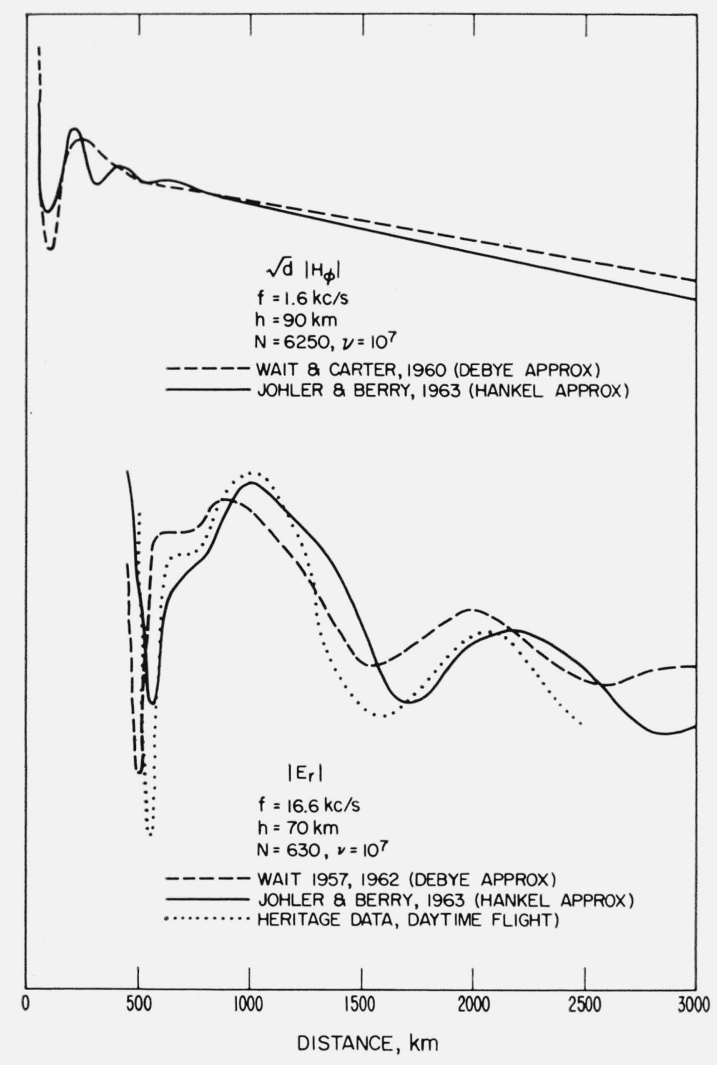

Figure 3. Comparison of fields computed with Debye and Hankel approximations.
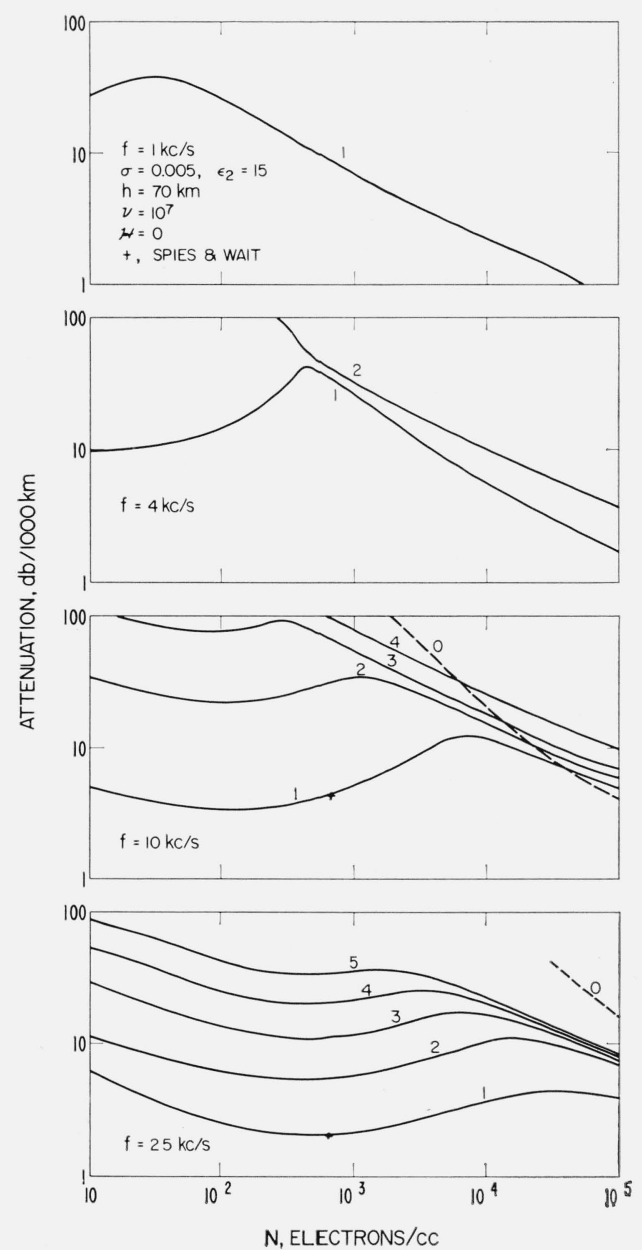

Figure 4. Attenuation rates of important modes as a function of the electron density of the model ionosphere, $\mathrm{N}$ electrons $/ \mathrm{cm}^{3}$.

function of electron density is sufficiently different from the behavior of the attenuation rate to suggest that the ambiguity in the preceding paragraph can be (at least partially) resolved by simultaneously considering the attenuation rate and phase velocity of the dominant mode.

As an example, a model was sought for the following case [Swanson, 1962]

$$
\begin{aligned}
& \text { frequency }=10.2 \mathrm{kc} / \mathrm{s}, \\
& \text { all sea path, daytime, } \\
& \text { attenuation rate }=3.5 \mathrm{db} / 1000 \mathrm{~km} \\
& \text { relative phase velocity }=1.0033 .
\end{aligned}
$$

Under the reasonable constraints, $h>60 \mathrm{~km},\left|\omega_{r}\right|<10^{6}$, successive approximations were computed until the above attenuation rate and phase velocity were attained. The resulting ionospheric parameters were then

$$
\begin{aligned}
& h=65 \mathrm{~km} \\
& \left|\omega_{r}\right| \cong 1.2 \times 10^{5} .
\end{aligned}
$$

No other values of $h$ and $\left|\omega_{r}\right|$ could be found which gave the required attenuation rate and phase velocity simultaneously. It is interesting to note that Wait deduced the values $h=70 \mathrm{~km}$, 


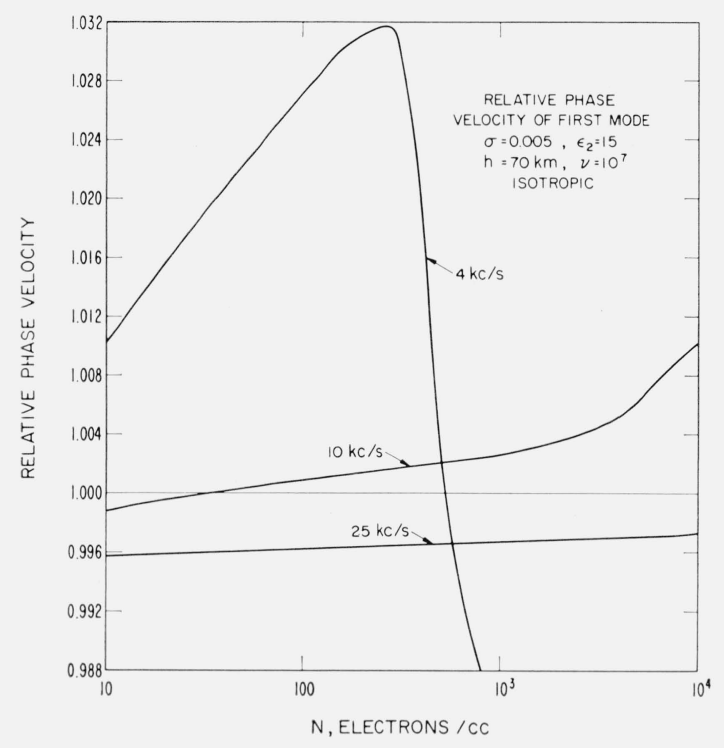

Figure 5. Relative phase velocity of first mode as a function of the electron density of the model ionosphere.

$\omega_{r}=1.2 \times 10^{5}$ for ELF propagation [Wait, 1962a] from an analysis of atmospheric wave forms.

The attenuation rate in the above example is approximate and different combinations of attenuation rate and phase velocity would yield different models. The important point is that simultaneous consideration of phase velocity and attenuation rate removed the ambiguity in extracting an effective model ionosphere from experimental data.

\section{Conclusions}

Careful derivation of the series of modes shows that the line integral represents waves that have traveled through the earth and reentered the airspace, and does not contribute to an ionosphere lateral wave for the homogeneous model ionosphere. This is satisfying since it agrees with the simple ray picture.

Quantitative estimates of the error incurred in using the Debye approximations were given.

The ambiguity which results from attempting to deduce an effective ionosphere from only the attenuation rate can be removed by considering attenuation rate and phase velocity simultaneously.

\section{References}

Bremmer, H. (1949), Terrestrial Radio Waves (Elsevier Publishing Co., New York).

Budden, K. G. (1961), The Wave-Guide Mode Theory of Wave Propagation (Prentice-Hall, Inc., New Jersey).

Fok, V. A. (1946), Diffraction of radiowaves around the earth's surface, (published Acad. of Science, USSR). Friedman, B. (1951), The theory of electromagnetic waves, A Symposium (Interscience Publishers, Inc.). Jahnke, E., and F. Emde (1945), Tables of Functions, 4th ed. (Dover Publications, New York).

Johler, J. R., and L. A. Berry (Nov.-Dec. 1962), Propagation of terrestrial radio waves of long wavelength, J. Res. NBS 66D (Radio Prop.), No. 6, 737-773.

Schumann, W. O. (1954), Uber die oberfelder bei der Ansbreitung langer, elektrischer wellen im system erdeluft-ionosphare und 2 anwendungen (horiyontaler und sunkrechter dipol), A. Angew. Phys. 6, 35-43.

Swanson, E. R. (1962), private communication.

Wait, J. R. (June 1957), The mode theory of VLF ionospheric propagation for finite ground conductivity, Proc. IRE 45, No. 6, 760-767.

Wait, J. R. (Mar.-Apr. 1960), Terrestrial propagation of very-low-frequency radio waves, J. Res. NBS 64D (Radio Prop.), No. 2, 153-204.

Wait, J. R. (1962a), Electromagnetic Waves in Stratified Media (Pergamon Press, Inc., New York). 
Wait, J. R. (July 1962b), Introduction to the theory of VLF propagation, Proc. IRE 50, No. 7, $1624-1648$. Wait, J. R. (1963), The mode theory of VLF radio propagation for spherical earth and concentric anistropic ionosphere, Can. J. Phys. 41, 199-315.

Wait, J. R., and N. F. Carter (Mar. 1960), Field strength calculations for ELF radio waves, NBS Tech. Note No. 52 .

Watson, G. N. (1918), The diffraction of electric waves by the earth, Proc. Roy. Soc. London $95,83$.

Watson, G. N. (1919), The transmission of electric waves round the earth, Proc. Roy. Soc. London 95 , 546.

(Paper 68D1-317) 